\title{
Induction of donor-specific tolerance to heart transplantation: From concept to clinical translation
}

Lillian Kang, MD, ${ }^{\mathrm{a}, \mathrm{b}}$ M. Louise Markert, MD, PhD, ${ }^{\mathrm{c}, \mathrm{d}}$ and Joseph W. Turek, MD, PhD, MBA ${ }^{\mathrm{a}, \mathrm{b}, \mathrm{e}}$

While significant progress has been made in the 54 years since the world's first heart transplant in 1967, the longevity of heart transplants is limited by rejection and the innumerable complications of chronic immunosuppression. Although immunosuppression regimens, when administered ideally, have evolved to offer reliable protection against most acute forms of rejection, they remain imperfect because of their lack of donor specificity, opportunistic infections, and significant medication toxicities. Instead, one of the goals that the field has been working toward is tolerance. Operational donor-specific tolerance requires 2 key features: The recipient does not reject the graft despite the absence of sustained immunomodulatory treatment and still retains the ability to mount adequate protective and regulatory immune responses. Tolerance has been considered the Holy Grail, because it would effectively eliminate the need for chronic immunosuppression and improve heart transplant longevity. Like the Grail, its existence is suspect, but the quest for it remains laudable. This review seeks to discuss the methods for inducing donor-specific tolerance in heart transplantation and their clinical potential.

\section{DRUG-BASED THERAPIES}

Methods of inducing donor-specific tolerance can be categorized as drug based, cell based, and mixed chimerism (Figure 1). The most prominent of the drug-based therapies is costimulation blockade.

\section{Costimulation Blockade}

As the primary cell involved in transplant rejection, most forms of costimulation blockade target T-cell activation. ${ }^{1}$ For a $\mathrm{T}$ cell to mount an immune response to a novel antigen, such as a polymorphic molecule on a transplanted heart, it must both recognize the antigen and then determine an appropriate response. Antigen recognition serves as a

\footnotetext{
From the ${ }^{\mathrm{a} D e p a r t m e n t}$ of Surgery, and ${ }^{\mathrm{b}}$ Duke Congenital Heart Research \& Training Laboratory, Duke University, Durham, NC; Departments of ${ }^{\mathrm{c}}$ Pediatrics and ${ }^{\mathrm{d}} \mathrm{Im}-$ munology, Duke University Medical Center, Durham, NC; and ${ }^{\mathrm{e} D u k e}$ Children's Pediatric \& Congenital Heart Center, Duke Children's Hospital, Durham, NC

Received for publication Sept 28, 2021; revisions received Dec 5, 2021; accepted for publication Dec 15, 2021; available ahead of print Jan 14, 2022.

Address for reprints: Joseph W. Turek, MD, PhD, MBA, Division of Cardiovascular and Thoracic Surgery, Department of Surgery, Duke University Medical Center, 2301 Erwin Rd, DUMC 3474, Durham, NC 27710 (E-mail: joseph.turek@duke. edu).

J Thorac Cardiovasc Surg 2023;165:1661-6

0022-5223/\$36.00

Copyright (c) 2022 by The American Association for Thoracic Surgery

https://doi.org/10.1016/j.jtcvs.2021.12.048
}

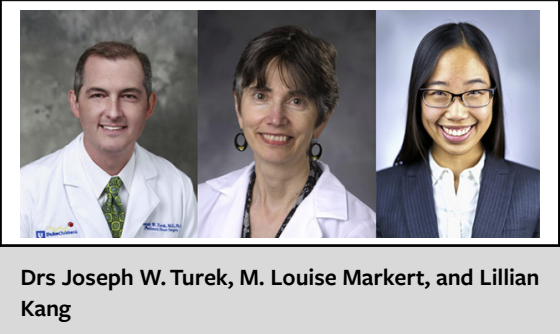

CENTRAL MESSAGE

Donor-specific tolerance for heart transplant can be influenced by costimulation blockade, cell-based targets, or mixed chimerism. Thymic interventions are poised to propel the field into translation.

See Commentary on page 1667. primary signal that identifies an antigen as worthy of a response. It manifests via interactions between the lymphocyte's antigen receptors - in the case of $\mathrm{T}$ cells, the T-cell receptor-with peptide-bound major histocompatibility complex (MHC) molecules. These can be allogeneic MHC molecules expressed directly by the graft or a donor peptide fragment presented indirectly by recipient antigen-presenting cells. This primary signal enables a decision that is governed by a second set of molecules, termed "costimulation molecules." The secondary signal derived from these molecules directs the T-cell's response. These molecules can be stimulatory (eg, CD28) or suppressive (eg, CD152), and the balance of these interactions determines the outcome of the immune recognition. Without a costimulatory signal, the default is to suppress any response. Thus, either stimulation of a suppressive molecule or blockade of a stimulatory molecule can prevent an aggressive novel antigen response. Lasting avoidance of specific immune responses can be achieved with episodic therapy. Clinically, blockade of proinflammatory costimulation signals after heart transplant mollifies graft-specific $\mathrm{T}$ cells.

In theory, T-cell clones rendered inactive via this method can foster donor-specific tolerance. In practice, several characteristics of costimulation have made it less effective 


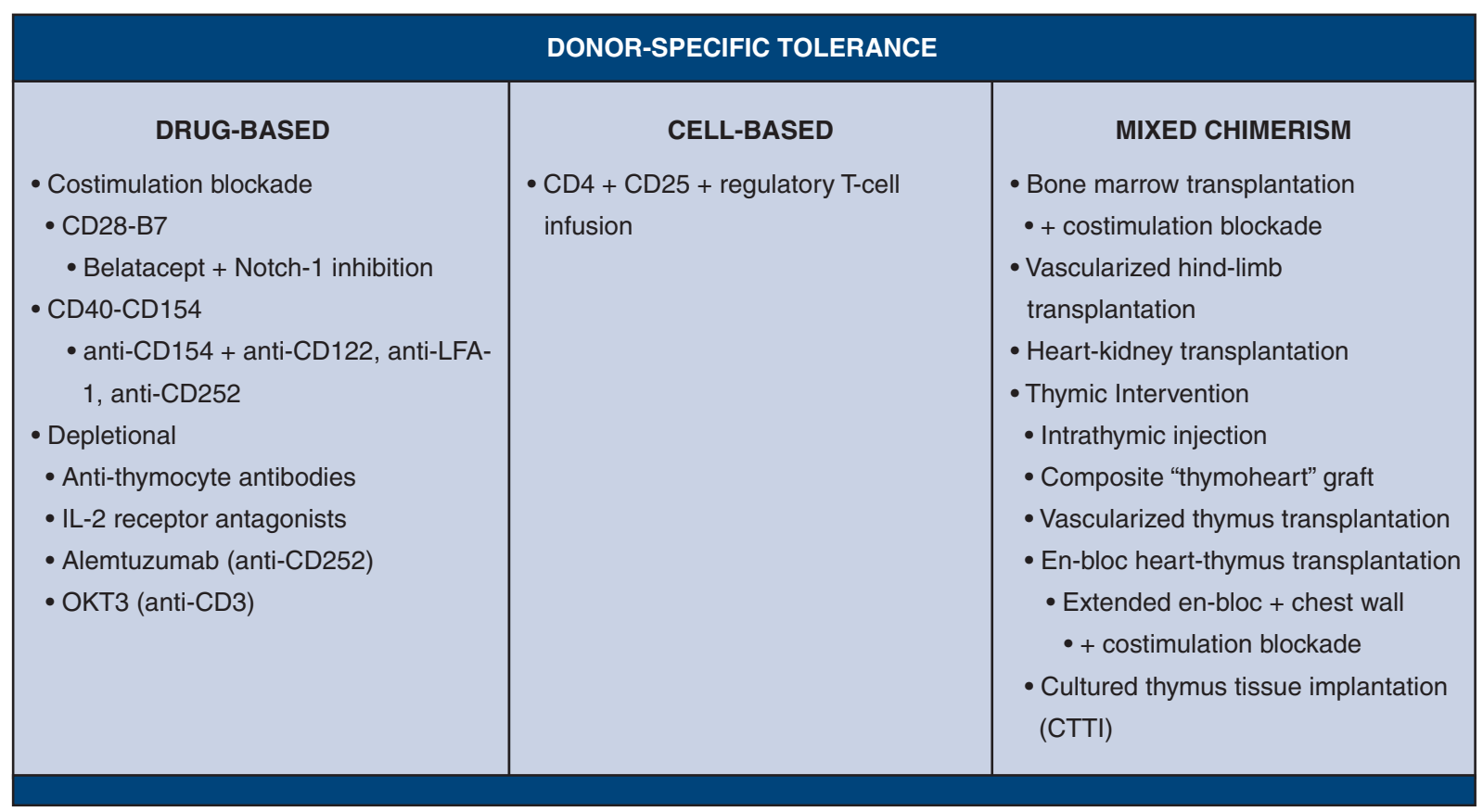

FIGURE 1. Mechanisms under investigation for inducing donor-specific tolerance in heart transplantation.

than experimental studies would suggest. The most important aspect of costimulation is that it is most relevant for initial encounters with new antigens. As cells gain antigen experience, memory $\mathrm{T}$ cells are reactivated solely by antigen recognition. Of note, memory $\mathrm{T}$ cells accumulate with age and are less responsive to many immunosuppressive agents, including T-cell depletion. ${ }^{2}$

Among the many costimulation signals, the 2 that have received the most attention are the CD28-B7 and the CD40-CD154 receptor ligand pairs. Blockade of these pairs was used together in prior mouse studies, demonstrating robust prolongation of allograft survival off immunosuppression. ${ }^{3}$ However, this effect was not sustained and failed to meet the tolerance threshold. Many other targets have subsequently been used in conjunction with CD40-CD154 antagonism. A combination of anti-CD154, CD122 (interleukin-2R subunit beta), lymphocyte function associated antigen-1 (CD11a/CD18 integrin), and CD252 (OX40 ligand) antibodies has been shown to prolong allograft survival in sensitized mice without immunosuppression to approximately 22 days post-transplantation. ${ }^{4}$ This treatment did not prevent chronic rejection and graft failure. Using costimulation blockade alone to induce sustained donor-specific tolerance has yet to reach the clinical applicability in heart transplantation.

Most recently, an approach in mice combined postoperative Notch-1 inhibition on the day of surgery and then every other day for 10 days with a single dose of post-transplant CD28/B7 antagonism via CTLA4-Ig, also known as "belatacept." ${ }^{5}$ Belatacept is the only clinically approved transplantation agent, but is only labeled for use in kidney transplantation. ${ }^{6}$ This approach resulted in prolonged allograft survival without immunosuppression and was able to demonstrate donor specificity as treated mice rejected third-party cardiac allografts. These studies are the most promising example of costimulation blockade for induction of sustainable donor-specific tolerance. Although selected nonhuman primate kidney recipients have been able to be ultimately maintained solely on belatacept monotherapy, the relatively higher rate of early acute rejections has hampered translation into heart transplantation. ${ }^{7}$ Given that allosensitization is a critical problem in heart transplantation, trials using belatacept for pretransplant desensitization are emerging. ${ }^{8}$ Presently, costimulation blockade is widely recognized for its tolerogenic properties, but this approach alone is insufficiently robust to induce a durable state of operational tolerance.

\section{Depletional Methods}

While depletional methods were pioneered in kidney transplantation using CRM9-CD3 in nonhuman primates, variable attempts have been made in heart transplantation. In mechanistic studies, depleting mature dividing $\mathrm{T}$ cells shortly after transplantation using a EpTK mouse model bred to express herpesvirus thymidine kinase in mature $\mathrm{T}$ cells, which are susceptible to ganciclovir-induced apoptosis, allowed for allograft survival without immunosuppression past the study end point of 100 days. ${ }^{10}$ Mixed lymphocyte reaction showed that mice retained immune response to both reintroduced donor and third-party 
antigens. Although this work is promising, it is not a therapy that offers direct clinical translation because of the risk of losing donor-specific tolerance due to the continued reactivity of recipients to donor antigens. Further clinical work with other depletional agents, such as antithymocyte antibodies, interleukin-2 receptor antagonists, alemtuzumab (humanized rat immunoglobulin-0G1 anti-CD252 monoclonal antibody), and OKT3 (a murine antihuman CD3-specific antibody), in human heart transplantation has shown associations with lower mortality and fewer rejection episodes, ${ }^{11}$ but there remains no evidence in heart transplantation that depletional methods are able to induce donor-specific tolerance.

\section{CELL-BASED STRATEGIES}

Another method for inducing donor-specific tolerance involves the introduction of specific immune cell populations, for example, augmentation of specific T-cell populations. Infusion of $\mathrm{CD} 4+\mathrm{CD} 25+$ regulatory $\mathrm{T}$ cells after sublethal radiation and immediately before heart transplantation in mice was able to prolong allograft survival without any peritransplant immunosuppression. ${ }^{12}$ Isogenic $\mathrm{T}$ cells showed a superior effect compared with allogenic $\mathrm{T}$ cells, which is the clinically applicable population. These mice were not challenged with third-party antigens; thus, no conclusions can be drawn on whether this method is capable of inducing donor-specific tolerance. Another limitation of this method is the requirement of preoperative radiation as well as donor T-cell isolation and infusion. Preoperative radiation would be possible but suboptimal; to isolate donor $\mathrm{T}$ cells and then infuse said $\mathrm{T}$ cells pretransplant, a donor would have to be maintained after harvesting $\mathrm{T}$ cells or the heart would have to be preserved for a prolonged period. With the advent of ex vivo perfusion technology, it is possible that donor organ cold storage duration may be prolonged eventually, but at present the feasibility of this approach is limited.

\section{MIXED CHIMERISM}

In heart transplantation, currently the most promising method of inducing donor-specific tolerance is mixed chimerism. Interventions using the principle of mixed chimerism aim to alter the recipient's immune system to include a mix of both native recipient and transplanted donor components such that donor antigens are accepted or tolerated as "self." Although this method was pioneered in the context of graft-versus-host disease for bone marrow transplantation (BMT) ${ }^{13}$ it was quickly identified as a promising method for inducing donor-specific tolerance across solid organ transplants as well. Based on successful forays into kidney transplantation, ${ }^{14}$ concurrent BMT with heart transplantation became a popular topic of investigation. Original studies in a dog model that received total lymphoid irradiation followed by BMT showed prolonged survival without immunosuppression, but this effect was not sustainable. ${ }^{15}$ It was also demonstrated that the dogs had global disruption of immune response such that they mounted no response to third-party antigens.

With the distinction of being one of the only methods translated into a clinical setting, concurrent BMT and heart transplantation were attempted with limited success in humans. Recipients who received MHC-mismatched BMT along with heart transplants had lower rates of acute cellular rejection and cardiac allograft vasculopathy than controls after steroids were weaned. ${ }^{16}$ In this model, steroids were weaned but immunosuppression was never discontinued entirely and donor specificity was not examined. Importantly, although chimerism was initially detectable, minimal chimerism was detectable in peripheral blood samples by 3 months post-transplantation. These results support the hypothesis that mixed chimerism, if achievable and self-sustaining, may be able to successfully induce donor-specific tolerance, but concurrent BMT alone is not likely a viable or durable method.

Other groups have combined concurrent BMT with costimulatory blockade. A mouse model with pretransplant BMT as well as CD28/B7 and lymphocyte function associated antigen-1 antagonism followed by skin transplantation 4 to 6 weeks after treatment and subsequently heart transplantation 2 weeks later showed induction of long-term donor-specific tolerance of heart allografts, but chronically rejected skin grafts of the same background as the BMT and heart. ${ }^{17}$ Although this brings both costimulation blockade and mixed chimerism via BMT closer to clinical relevance, the requirement of receiving a BMT and costimulation treatment 1 to 2 months before a heart transplant remains a significant limitation.

In a similar vein, vascularized hind-limb and heart cotransplantation has been shown to induce donor-specific tolerance in rats. ${ }^{18}$ Rats in these studies received orthotopic vascularized hind-limb transplants followed by weaning of immunosuppression, skin grafts, and then heterotopic heart transplants. Despite discontinuation of immunosuppression, rats were able to accept skin grafts and heart transplants from the same donor background as the hind limb transplants and were able to reject third-party skin grafts, demonstrating donor-specific tolerance. Of note, 6 to 7 weeks after discontinuation of immunosuppression, chimerism was no longer detectable in the peripheral blood, but the animals were still able to demonstrate donor-specific tolerance. Mixed chimerism in this case is due to the introduction of the donor hindlimb bone marrow. Although this method is not a candidate for clinical translation, it is a method that has been shown to induce durable donor-specific tolerance for heart transplantation. 
Although experience in human heart-kidney co-transplantation has not resulted in donor-specific tolerance, many studies in swine and mice have shown this phenomenon. ${ }^{19,20}$ Earlier authors hypothesized that heart-kidney co-transplantation in swine was able to induce allograft tolerance due to transfer of donor kidney regulatory $\mathrm{T}$ cells and kidney-derived cells trafficking to the host thymus to induce mixed chimerism. Further elucidation into the cellular underpinnings in mouse models showed that depletion of regulatory $\mathrm{T}$ cells caused tolerance deterioration with allograft rejection, further strengthening this reasoning. Unfortunately, although this method does allow for further mechanistic understanding of the immunology of donor-specific tolerance, it does not hold any direct clinical applicability.

\section{Thymic Intervention}

Before thymic transplantation, a variety of groups attempted to use intrathymic injection in rats as a method for establishing mixed chimerism. The first wave consisted of differing preparations of donor splenocytes injected into the thymus pretransplantation. ${ }^{21,22}$ This method has been shown to prolong survival without immunosuppression compared with controls and to achieve full donor-specific tolerance when paired with T-cell depletion via intraperitoneal administration of antilymphocyte serum. In mice, intrathymic injection of anti-Fas antibodies at the time of transplantation resulted in thymocyte apoptosis and allowed for prolonged allograft survival off immunosuppression, but studies did not show donor-specific tolerance. ${ }^{23}$

An alternative approach to establishing durable mixed chimerism is transplantation of composite "thymoheart" grafts. ${ }^{24}$ Thymohearts were prepared by injecting minced donor thymus into the donor right atrial appendage and cardiac fat pads 60 to 90 days before transplantation. Swine with thymoheart transplants were able to develop donorspecific tolerance. In a theme similar to a majority of these methods, thymohearts are not easily translated into the clinical setting because they require a 60 - to 90 -day pretransplant donor intervention.

Promise in the thymoheart realm prompted investigations into co-transplantation with vascularized thymus. ${ }^{25}$ After weaning immunosuppression 4 weeks post-transplant in swine, this method was able to prolong short-term allograft survival, but still resulted in rejection between 80 and 110 days postoperatively. Despite its potential ease of introduction into the clinical realm, vascularized thymus cotransplantation has not been shown to be an effective means of inducing donor-specific tolerance.

As a response, studies pivoted to an en bloc method. This method proved promising with en bloc heart/thymus transplanted miniature swine demonstrating prolonged graft survival to the study end point of 200 days after discontinuation of immunosuppression. ${ }^{26}$ Despite seemingly indefinite allograft survival off immunosuppression, the donor specificity of the tolerance with third-party antigen challenge was not examined. Combining this method with concepts from the vascularized hind-limb transplant as a source of donor bone marrow, one variation of the en bloc method included en bloc heart, thymus, and chest wall. ${ }^{27}$ Mice who received these extensive en bloc transplants also showed prolonged survival off immunosuppression. Survival was augmented by the addition of costimulation blockade with antiCD154 antibodies. Neither en bloc method has demonstrated donor-specific tolerance nor retention of native immune response to third-party antigens. While en bloc heart/thymus transplants pose potential for clinical translation, extended en bloc transplantations are practically limited.

Although composite thymoheart and en bloc heart/ thymus transplants have fallen short, a separate nontransplantation method known as "cultured thymus tissue implantation" [CTTI] shows promise. In CTTI, donor thymus is thinly sliced and cultured on nitrocellulose filters with fresh media dripped across sections before implantation. This step separates CTTI from other approaches because freshly explanted donor thymus contains a high burden of T cells, which may result in graft-versus-host disease if transplanted directly. Culturing donor thymus in this manner is advantageous on multiple fronts. It results in effective donor T-cell depletion, facilitates changes that maximize localization of recipient T-cell precursors to donor thymus, and provides niches to support recipient T-cell development and maturation within the retained network of donor thymic epithelium and stromal cells. ${ }^{28}$ After culture, slices of thymus tissue are implanted into furrows between muscle bundles within the quadriceps of patients as nonvascularized grafts $^{29}$ (Figure 2). Clinically, CTTI has been used safely and successfully to treat children with DiGeorge's anomaly. ${ }^{30}$ When combined with recipient

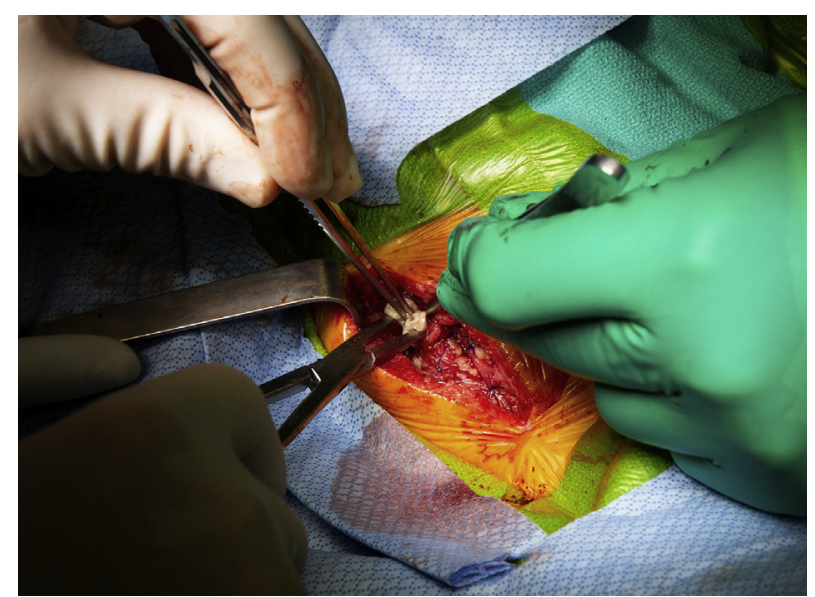

FIGURE 2. Implantation of culture thymus tissue in the quadriceps muscle. 
thymectomy and heart transplantation in rats, pretransplant CTTI allows for donor-specific tolerance off immunosuppression and rejection of third-party heart transplants. ${ }^{31}$

\section{Clinical Translation}

In August of 2021 at our institution, under a Food and Drug Administration Investigational New Drug application and with Food and Drug Administration compassionate care approval, we performed the world's first orthotopic heart transplant (OHT) in an infant followed by CTTI from the same donor. Because of the patient's heart failure and T-cell deficiency of undetermined etiology, the patient was an ideal candidate to undergo OHT and completion thymectomy followed by CTTI, because CTTI has already been proven efficacious in treating patients with thymic insufficiency. Monthly quantification of naïve T-cell populations via flow cytometry will allow us to assess whether the patient is able to demonstrate immune reconstitution, at which time we will begin considerations of weaning immunosuppression. As we await outcomes from this case, it will be important to pursue ongoing studies to further validate concurrent CTTI and OHT in humans. Research is needed to continue to optimize pre-CTTI T-cell depletion in immunocompetent patients. The efficacy of CTTI in human infants with congenital athymia at less than 9 months of age has been thoroughly demonstrated. However, because of the natural course of thymic involution with age, it will be important to determine the upper age limit of thymic viability for CTTI. Ongoing research and future clinical trial results support further clinical translation of CTTI to human patients. Additionally, further studies must be undertaken to achieve the goal of shipping cultured thymus tissue to other centers for implantation. Together, further investigations will provide additional information regarding the clinical potential of concurrent CTTI as a method for inducing durable donor-specific tolerance in heart transplantation.

\section{CONCLUSIONS}

The coveted goal of donor-specific tolerance in heart transplantation has spurred a multitude of investigations. From costimulation blockade with variations of CD154 antagonism to a plethora of cell-based strategies including the manipulation of $\mathrm{T}$ cells and dendritic cells to diverse approaches of establishing mixed chimerism with concurrent BMT and vascularized hind-limb transplant, each of these therapies has progressed the field closer to the target. As it stands, the field of heart transplantation is on the brink of clinical translation with advances in thymic interventions. While vascularized thymus co-transplantation has fallen short, and composite thymoheart transplant will likely not become clinically applicable, further work interrogating the details of en bloc heart/thymus transplantation and CTTI holds the potential for advancing such methods of inducing durable donor-specific tolerance into the translational domain and eventually into the clinical realm.

\section{Conflict of Interest Statement}

CTTI, RVT-802, is an investigational product implanted in patients under an Investigational New Drug application with the Food and Drug Administration. M.L.M. is the "sponsor" of the investigations and developed the technology for CTTI. Duke University has licensed the technology to Enzyvant Therapeutics GmbH. There are 2 licenses, one for CTTI in athymic patients and the second for use of CTTI, to induce tolerance in solid organ transplantation. M.L.M. and Duke University have received royalties from Enzyvant. Portions of M.L.M.'s and her research team's salaries are being paid by funding from Enzyvant. If the technology is commercially successful in the future, M.L.M and Duke University may benefit financially. The salary and other items needed to create CTTI are paid at cost by insurance. All other authors reported no conflicts of interest.

The Journal policy requires editors and reviewers to disclose conflicts of interest and to decline handling or reviewing manuscripts for which they may have a conflict of interest. The editors and reviewers of this article have no conflicts of interest.

\section{References}

1. Larsen CP, Knechtle SJ, Adams A, Pearson T, Kirk AD. A new look at blockade of T-cell costimulation: a therapeutic strategy for long-term maintenance immunosuppression. Am J Transplant. 2006;6:876-83.

2. Pearl JP, Parris J, Hale DA, Hoffmann SC, Bernstein WB, McCoy KL, et al Immunocompetent T-cells with a memory-like phenotype are the dominant cell type following antibody-mediated T-cell depletion. Am J Transplant. 2005;5:465-74.

3. Pierson RN III, Chang AC, Blum MG, Blair KS, Scott MA, Atkinson JB, et al. Prolongation of primate cardiac allograft survival by treatment with ANTICD40 ligand (CD154) antibody. Transplantation. 1999;68:1800-5.

4. Xie B, Chen J, Wang F, Lan T, Wang Y, Xia J, et al. Monoclonal antibody treatment to prolong the secondary cardiac allograft survival in alloantigen-primed mice. Scand J Immunol. 2010;71:345-52.

5. Magee CN, Murakami N, Borges TJ, Shimizu T, Safa K, Ohori S, et al. Notch-1 inhibition promotes immune regulation in transplantation via regulatory $\mathrm{T}$ cell dependent mechanisms. Circulation. 2019;140:846-63.

6. Kirk AD, Adams AB, Durrbach A, Ford ML, Hildeman DA, Larsen CP, et al. Optimization of de novo belatacept-based immunosuppression administered to renal transplant recipients. Am J Transplant. 2021;21:1691-8.

7. Schmitz R, Fitch ZW, Xu H, Ghali A, Mehta AK, Guasch A, et al. Kidney transplantation using alemtuzumab, belatacept, and sirolimus: five-year follow-up. Am J Transplant. 2020;20:3609-19.

8. Alishetti S, Farr M, Jennings D, Serban G, Uriel N, Sayer G, et al. Desensitizing highly sensitized heart transplant candidates with the combination of belatacept and proteasome inhibition. Am J Transplant. 2020;20:3620-30.

9. Armstrong N, Buckley P, Oberley T, Fechner J Jr, Dong Y, Hong X, et al. Analysis of primate renal allografts after T-cell depletion with anti-CD3-CRM9. Transplantation. 1998;66:5-13.

10. Braunberger E, Raynal-Raschilas N, Thomas-Vaslin V, Bruneval P, Fabiani JN, Carpentier A, et al. Tolerance induced without immunosuppression in a T-lymphocyte suicide-gene therapy cardiac allograft model in mice. $J$ Thorac Cardiovasc Surg. 2000;119:46-51.

11. Tzani A, Van den Eynde J, Doulamis IP, Kuno T, Kampaktsis PN, Alvarez P, et al Impact of induction therapy on outcomes after heart transplantation. Clin Trans plant. 2021;35:e14440. 
12. Lu X, Yang X, Zhou Q, Wang Y, Feng S, Jin J, et al. Cardiac allograft tolerance induced by isogeneic CD4+CD25+ regulatory T cells. Exp Clin Transplant. 2014;12:133-8.

13. Mapara MY, Kim YM, Wang SP, Bronson R, Sachs DH, Sykes M. Donor lymphocyte infusions mediate superior graft-versus-leukemia effects in mixed compared to fully allogeneic chimeras: a critical role for host antigenpresenting cells. Blood. 2002;100:1903-9.

14. LoCascio SA, Morokata T, Chittenden M, Preffer FI, Dombkowski DM, Andreola G, et al. Mixed chimerism, lymphocyte recovery, and evidence for early donor-specific unresponsiveness in patients receiving combined kidney and bone marrow transplantation to induce tolerance. Transplantation. 2010; 90:1607-15.

15. Kahn DR, Dufek JH, Hong R, Caldwell WL, Thomas FJ, Kolenda DR, et al. Heart and kidney transplantation using total lymphoid irradiation and donor bone marrow in mongrel dogs. J Thorac Cardiovasc Surg. 1980;80:125-8.

16. Pham SM, Rao AS, Zeevi A, Kormos RL, McCurry KR, Hattler BG, et al. A clinical trial combining donor bone marrow infusion and heart transplantation: intermediate-term results. J Thorac Cardiovasc Surg. 2000;119:673-81.

17. Pilat N, Sabler P, Klaus C, Mahr B, Unger L, Hock K, et al. Blockade of adhesion molecule lymphocyte function-associated antigen-1 improves long-term heart allograft survival in mixed chimeras. J Heart Lung Transplant. 2018;37:1119-30.

18. Liu Q, Wang Y, Nakao A, Zhang W, Gorantla V, Zheng XX. Heart allograft tolerance induced and maintained by vascularized hind-limb transplant in rats. Clin Dev Immunol. 2013;2013:483856.

19. Yamada K, Mawulawde K, Menard MT, Shimizu A, Aretz HT, Choo JK, et al. Mechanisms of tolerance induction and prevention of cardiac allograft vasculopathy in miniature swine: the effect of augmentation of donor antigen load. $J$ Thorac Cardiovasc Surg. 2000;119:709-19.

20. Yang C, Ge J, Rosales I, Yuan Q, Szuter E, Acheampong E, et al. Kidney-induced systemic tolerance of heart allografts in mice. JCI Insight. 2020;5:e139331.

21. Shen Z, Mohiuddin M, Goldstein C, Yokoyama H, DiSesa VJ. Durability of donor-specific and organ-specific heart transplant tolerance induced by intrathymic pretreatment with allogeneic spleen cells. J Thorac Cardiovasc Surg. 1996; 111:429-31.
22. Shen Z, Kline G, Mohiuddin M, DiSesa VJ. Histocompatibility differences and cardiac transplant tolerance produced by intrathymic pretreatment. J Thorac Cardiovasc Surg. 1994;107:1472-5.

23. Djamali A, Waller KR, McAnulty J, Hullett D, Becker BN, Odorico JS. Intrathymic injection of anti-Fas monoclonal antibody prolongs murine non-vascularized cardiac allograft survival. Transpl Int. 2004;17:301-9.

24. Menard MT, Schwarze ML, Allan JS, Johnston DR, Mawulawde K, Shimizu A, et al. Composite "thymoheart" transplantation improves cardiac allograft survival. Am J Transplant. 2004;4:79-86.

25. Nobori S, Samelson-Jones E, Shimizu A, Hisashi Y, Yamamoto S, Kamano C, et al. Long-term acceptance of fully allogeneic cardiac grafts by cotransplantation of vascularized thymus in miniature swine. Transplantation. 2006;8: 26-35.

26. Johnston DR, Muniappan A, Hoerbelt R, Guenther DA, Shoji T, Houser SL, et al. Heart and en-bloc thymus transplantation in miniature swine. J Thorac Cardiovasc Surg. 2005;130:554-9.

27. Oh B, Furtmüller GJ, Malek V, Fryer ML, Brayton C, Walczak P, et al. Split tolerance in a murine model of heterotopic en bloc chest wall transplantation. Plast Reconstr Surg Glob Open. 2017;5:e1595.

28. Markert ML, Gupton SE, McCarthy EA. Experience with cultured thymus tissue in 105 children. J Allergy Clin Immunol. August 3, 2021; https://doi.org/10.1016/ j.jaci.2021.06.028

29. Rice HE, Skinner MA, Mahaffey SM, Oldham KT, Ing RJ, Hale LP, et al. Thymic transplantation for complete DiGeorge syndrome: medical and surgical considerations. J Pediatr Surg. 2004;39:1607-15.

30. Gupton SE, McCarthy EA, Markert ML. Care of children with DiGeorge before and after cultured thymus tissue implantation. J Clin Immunol. 2021;41:896-905.

31. Kwun J, Li J, Rouse C, Park JB, Farris AB, Kuchibhatla M, et al. Cultured thymus tissue implantation promotes donor-specific tolerance to allogeneic heart transplants. JCI Insight. 2020;5:e129983.

Key Words: cultured thymus tissue implantation, donorspecific tolerance, heart transplantation 